\title{
The Novel Anti-tumor Therapy Targeting the "Functional" Cancer Stem Cell Markers
}

\section{Go J Yoshida* and Hideyuki Saya}

Division of Gene Regulation, Institute for Advanced Medical Research, School of Medicine, Keio University, 35 Shinanomachi, Shinjuku-ku, Tokyo 160-8582, Japan

\begin{abstract}
It has recently been reported that molecular targeting drugs fail to overcome the "oncogene/oncogenic signaladdiction" of cancer cells. Tumor tissue is composed of heterogeneous cancer cells, so that the therapeutic response is difficult to predict. Recent advance in cancer research has strongly suggests that cancer stem cells contribute to the formation and maintenance of the heterogeneous cellular society in the tumor tissue. After all, this heterogeneity is the major cause of the acquired resistance to anti-tumor therapies. In this commentary, we would like to briefly explain the promising therapeutic targets of CD44 variant isoform and EpCAM, the "functional" cancer stem cell markers.
\end{abstract}

Keywords: Cancer stem cell; Heterogeneity; Stemness; CD44; xCT transporter; Redox stress; EpCAM; Nutrient microenvironment

\section{Tumor Heterogeneity and Cancer Stem Cells}

Tumor tissue is composed not only cancer cells but also various types of cells in the stroma; Cancer-associated fibroblasts (CAF), tumor-associated macrophages (TAM) etc. Cancer cells show invasion and metastasis in collaboration with CAF and TAM. Accumulating evidence suggests that tumor cells educate stroma cells as they enhance the malignant potential of tumor cells [1]. Given that cancer cells frequently have mutations in the cell-cycle regulatory proteins, tumor cells are mistaken as homogeneous. However, there exists a cellular heterogeneity in tumor tissues [2], which is why therapeutic response to the molecular targeting drugs is different depending on the genetic or epigenetic background of each tumor cell. Thus, the high degree of heterogeneity renders tumor tissue more easily to acquire the resistance to the molecular-targeting therapies focusing on "oncogene/oncogenic signal-addiction" [3]. Only the resistant clones survive, so that the level of heterogeneity is transiently diminished by the anti-cancer treatment, which is called "bottleneck effect". Generally speaking, this "bottleneck effect" is lost and the heterogeneity is again recognized in the recurrent tumor tissue [2].

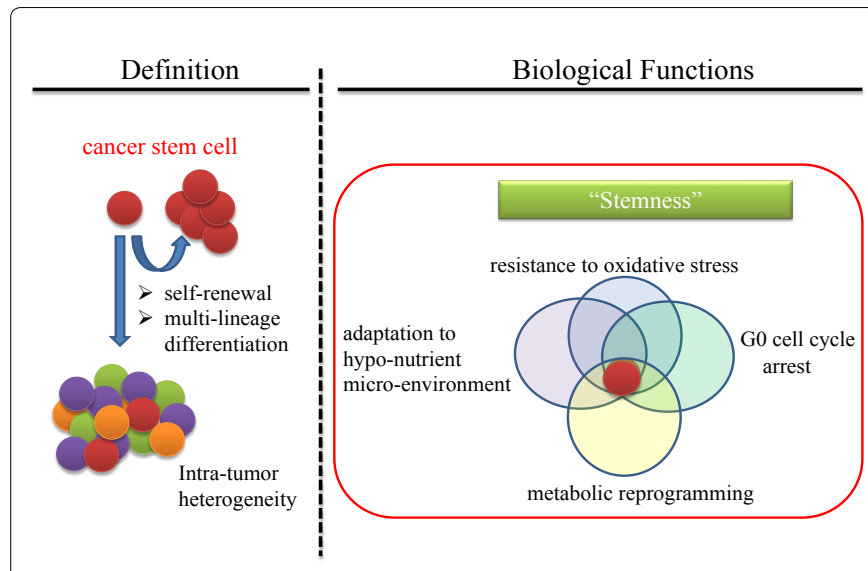

Figure 1: Bilateral perspectives into the entity of cancer stem cells (CSCs). CSCs are defined to show the self-renewal and multi-lineage differentiation potential, which contribute to the formation of the heterogeneous cellular population in the tumor tissue. On the other hand, CSCs have the biological characteristics to maintain the "stemness"; resistance to ROS or DNA damage, adaptation to hypo-nutrient or growth factor microenvironment and metabolic reprogramming. It still remains elusive, however, these two cell populations are exactly the same entity each other.
An emerging concept of the cancer stem cells (CSCs) simply explains how the hierarchy of tumor cells is formed. CSCs have the selfrenewal ability and multi-lineage differentiation potential [4,5], both of which are necessary to architect the secondary heterogeneous tumor lesion from a single cell (Figure 1). This diversity is responsible for the formation of minimal residual disease (MRD) [2], which leads to the latent relapse and the distant metastasis. MRD is frequently enriched in therapy-resistant clones with the "stemness" [6-8].

\section{The Significance of CSC in Terms of Therapeutic Strategy}

CSCs have been well-defined and intensively researched, but much remains to be elucidated about the function of CSCs in the tumor development and metastasis. We would like to focus on the biological characteristics of CSCs, which contributed to the therapeutic resistance. Stemness is composed of the various phenotypes of CSCs; resistance to redox stress via the synthesis of glutathione (GSH) and G0 cell cycle arrest under hypo-nutrient or growth factor conditions (Figure 1). Better understanding of the regulatory system of "stemness" is critical to develop the novel anti-tumor therapy focusing on CSCs.

Epithelial-mesenchymal transition (EMT) has widely been recognized as a crucial step in the invasion and metastasis as well as normal tissue development and wound healing [9]. EMT has long been believed to increase the number of CSCs at the invasive front and metastatic foci $[10,11]$.

CD44 is an adhesion molecule to extracellular matrix such as osteopontin and hyaluronic acids [12]. CD44 has a numerous isoforms because of alternative splicing machinery mainly regulated by RNA binding protein epithelial splicing regulatory protein 1 (ESRP1) and epigenetic modulation of the HistoneH3lysine9trimethylation (H3K9me3) [13-15]. While CD44 variant isoform (CD44v) is

*Corresponding author: Go J Yoshida, Division of Gene Regulation, Institute for Advanced Medical Research, School of Medicine, Keio University, 35 Shinanomachi, Shinjuku-ku, Tokyo 160-8582, Japan, Tel: +81-3-5363-3981; Fax: +81-3-5363-3982; E-mail: medical21go@yahoo.co.jp; hsaya@a5.keio.jp

Received December 17, 2013; Accepted January 08, 2014; Published January 15,2014

Citation: Yoshida GY, Saya H (2014) The Novel Anti-tumor Therapy Targeting the "Functional" Cancer Stem Cell Markers. Clin Exp Pharmacol 4: 147. doi:10.4172/2161-1459.1000147

Copyright: (c) 2014 Yoshida GY, et al. This is an open-access article distributed under the terms of the Creative Commons Attribution License, which permits unrestricted use, distribution, and reproduction in any medium, provided the original author and source are credited. 


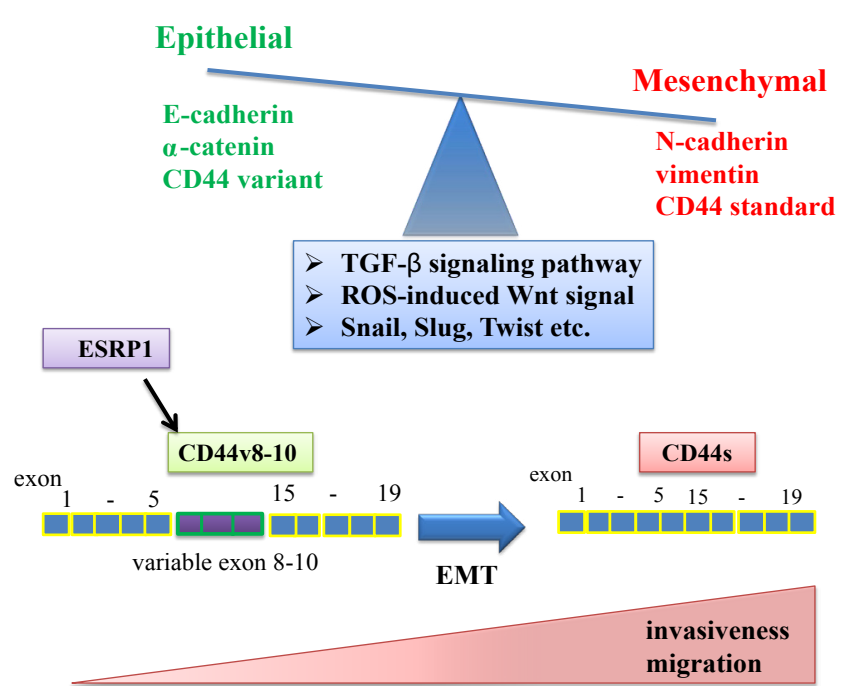

Figure 2: Redox stress-induced Wnt activation and EMT. ROS stimuli-induced Wnt activation, TGF- $\beta$ signaling, and EMT-inducing transcriptional factors such as Snail, Slug, Twist etc. enhance the invasive and migratory phenotype with the cadherin switch and the change in the alternative splicing of CD44. The splicing of CD44 mRNA is regulated by ESRP1, the expression of which is influenced by epigenetic changes.

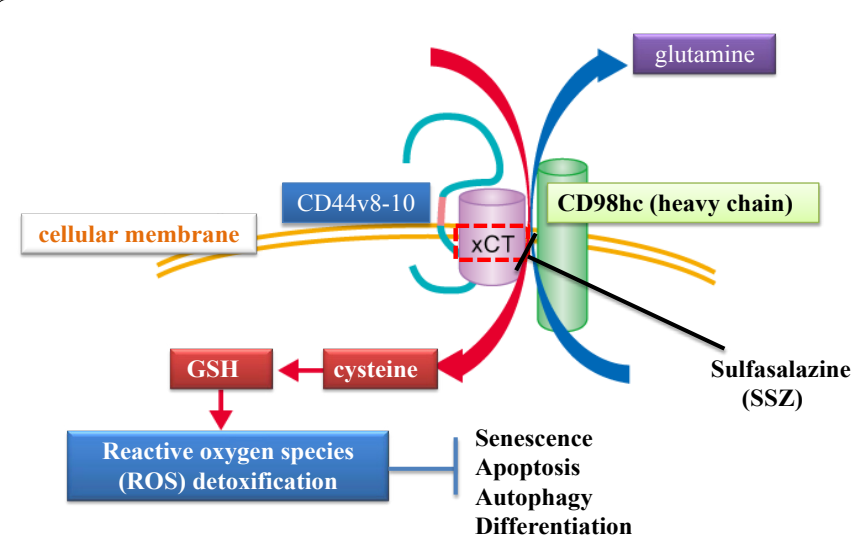

Figure 3: CD44v-xCT-GSH axis in the protection from redox stress. CSCs highly express CD44v8-10, which stabilizes XCT (cystine/glutamine) exchanging transporter, at the cellular membrane. xCT increases intracellular cysteine concentration, which leads to enhanced GSH synthesis. CSCs maintain ROS under the low level by the promoted GSH synthesis, thereby inhibiting senescence, apoptosis and autophagy. Sulfasalazine, a specific $\mathrm{xCT}$ inhibitor, collapses CD44v-xCT-GSH axis to regulate the redox stress in CSCs.

predominantly expressed in epithelial cancer cells, the CD44 standard isoform (CD44s) is mainly expressed in mesenchymal cancer cells. Epithelial tumor cells no longer express CD44v after EMT, and instead, express CD44s with high migratory and invasive phenotypes [13] (Figure 2). Mesenchymal tumor cells with CD44s expression after EMT tend to show the activation of phosphatidylinositol-3 kinase (PI3K)/ Akt signaling pathway in the metastatic foci in the lungs of the breast cancer cells [13].

Several transcription factors have been identified to induce EMT; FOXC2 in the basal-like breast cancer [16], SIP1/ZEB2 in ovarian, breast, and hepatic tumors [17], and Snail, Slug, Twist in several kinds of cancers [9]. Contrary to the accepted wisdom, the homeobox transcriptional factor Prrxl induces EMT but decreases the number of CSCs. mesenchymal-epithelial transition (MET) is an important step for cancer cells to colonize and proliferate at the pre-metastatic niche. That is why the knockdown of Prrx 1 induces MET and promotes the lung metastasis of breast cancer cells [18].

Furthermore, it has been demonstrated that tumor cells maintain epithelial phenotype with the high expression of CD44v at the invasive front [10]. This seems paradoxical to the current concept, but some researcher insist on the presence of CAFs with CSCs at the invasive front [19]. Indeed, CD44v is strongly expressed at the invasive front and negatively correlated with the expression of oncogenic protein c-Myc. Redox stress-induced Wnt signal activation is responsible for the inversed relationship between CD44v and c-Myc [10]. The irreversible quiescence of CSCs is finely regulated by the modulation of several kinds of stress derived from tumor microenvironment.

\section{Novel Therapy Attacking the "Functional" CSC Markers}

There are many molecules exclusively expressed in CSCs. The specific isoform of CD44 and EpCAM are positively correlated with each other even in normal epithelial cells [20]. However, both the CD44v and Epithelial Cell Adhesion Molecule (EpCAM) are not just the surface markers of CSCs. We would like to briefly introduce the recent findings on this therapeutic approach.

\section{Novel therapy attacking the robustness against oxidative stress of CSCs}

The heterogeneous expression pattern of CD44 is caused by the heterogeneous expression of ESRP1, which is mainly responsible for the alternative splicing of CD44 [13,15]. Epigenetic modulation of the histone at the site of ESRP1 is dynamic depending on the tumor microenvironment, which strongly supports the idea of "dynamic stemness model" [21], which is contrary to the widely-accepted idea of CSC hierarchy model. CD44v-positive cells are not necessarily express CD44v. After all, the H3K4me3 of the ESRP1 promoter lesion promotes ESRP1-inducing CD44v expression, whereas H3K27me3 suppresses it [15]. Intra-tumor heterogeneity in terms of CD44v expression is considered to be determined by this dynamic epigenetic change (Figure 2).

There are several isoforms of CD44v, depending on which variable exons are inserted. Furthermore, different isoforms of CD44v exhibit the different biological function and it has been suggested that CD44 variant is a promising therapeutic-target in the cancer therapy [22]. In particular, CD44v8-10, CD44 variant isoform including the variable exon 8-10, stabilizes xCT transporter at the cellular membrane. $\mathrm{xCT}$ forms a heterodimer with CD98 heavy chain (CD98hc), also referred to as $4 \mathrm{~F} 2$. $\mathrm{xCT}$ amino acid transporter help CSCs exchange glutamate and cystine [23]. Cysteine, which is converted from cysteine, is a scarce substrate of GSH, major anti-oxidant molecule. Cancer cells are exposed to excessive amount of reactive oxygen species (ROS), which induces apoptosis, autophagy, cellular senescence, and differentiation [24-26] (Figure 3). GSH works as a gatekeeper for CSCs to prevent the ROS accumulation and maintain the "stemness". This CD44v-xCTGSH axis enables CSCs to survive and proliferate under the redox stress conditions.

Importantly enough, xCT-GSH axis is not limited to tumors of epithelial tissues; glioma cells are reported to express $\mathrm{xCT}$ without $\mathrm{CD} 44 \mathrm{v}$ and the secreted glutamate is the cause of glioma-associated brain edema [27]. Besides, triple negative breast cancer tissues, pathologically classified into basal-typed and caludin (low)-typed 
mesenchymal tumors, highly express xCT and show the "glutamine addiction" independently of CD44v expression [28]. Sulfasalazine (SSZ), an anti-inflammatory drug long used for patients with ulcerative colitis, has been shown to prevent the function of $\mathrm{xCT}$ transporter $[15,23,28,29]$ and expected to break down the robustness of CSCs against redox stress (Figure 3).

\section{Novel therapy attacking the sensitivity to nutrient microenvironment of CSCs}

EpCAM is expressed in the circulating tumor cells (CTCs) in the blood and several neutralizing antibodies have been developed for the prevention and the treatment for tumor metastasis [30,31]. For instance, catumaxomab is the antibody preparation against human EpCAM and CD3 and clinically effective for the patients with advanced ovarian tumors with recurrent symptomatic malignant ascites [32]. However, it still remains unknown how EpCAM contributes to the survival potential of CSCs under oppressive conditions.

In the perspective of membrane-molecular function, EpCAM has recently been identified as a sensor of growth factor microenvironment by the formation of super-complex with amino-acid transporters [33,34]. For example, EpCAM is positively correlated with the expression and localization of amino acid transporter L-type amino acid transporter (LAT) 1 promotes the uptake of leucine, thereby enhancing mammalian target of rapamycin (mTOR) signaling pathway $[33,35]$. There exists a crosstalk between mTOR and 5'-Adenosine monophosphate-activated protein kinase (AMPK) signal pathways. In the steady state with enough amount of energy, EpCAM-low or-negative cancer cells tend to be activated as compared with EpCAM-high CSCs. Serum starvation leads to AMPK signal activation exclusively in CSCs in the specific type of prostate cancers [33]. In other words, EpCAM exists as an upstream molecule for AMPK signal pathway to sense the change of the nutrient microenvironment by the formation of super-complex with amino acid transporters in cooperation with CD147 (EMMPRIN) and CD98hc (the chaperon referred to as $4 \mathrm{~F} 2$ ) [34].

Thus, it is highly likely that EpCAM expression makes CSCs sensitive to the change in glucose and growth factors by the regulation of the localization and stabilization of several monocarboxylate and amino acid transporters. Prostate cancer cells respond to lack of growth factors differently depending on the EpCAM expression amount [33]. EpCAM-positive CSCs may rapidly adapt to the change in nutrient microenvironment. Long-term serum starvation promotes cell cycle arrest and the acquisition of a quiescent phenotype, often observed in CTCs. EpCAM-high cancer cells entered the G0 phase via the SCF (Fbw7)-c-Myc axis, while EpCAM-low cells via the Skp2-p27 axis. EpCAM enhances the ability of cells to become dormant under long-term serum starvation and promotes cell cycle re-entry from this dormancy upon exposure to growth factors. Thus, acquisition of dormancy is an important factor of the biological feature of CSCs induced by EpCAM expression.

\section{Conclusion}

We have briefly introduced the importance of the therapeutic strategy targeting the "functional" CSC markers. We strongly hope the advancement of the research and therapeutic strategy by uncovering how the "stemness" is maintained during the invasion and metastasis by those molecules.

\section{References}

1. Casazza A, Di Conza G, Wenes M, Finisguerra V, Deschoemaeker S et al. (2013) Tumor stroma: a complexity dictated by the hypoxic tumor microenvironment. Oncogene.
2. Meacham CE, Morrison SJ (2013) Tumour heterogeneity and cancer cell plasticity. Nature 501: 328-337.

3. Sharma SV, Settleman J (2007) Oncogene addiction: setting the stage for molecularly targeted cancer therapy. Genes Dev 21: 3214-3231.

4. Clarke MF, Dick JE, Dirks PB (2006) Cancer stem cells--perspectives on current status and future directions: AACR Workshop on cancer stem cells. Cancer research 66: 9339-9344.

5. Bonnet D, Dick JE (1997) Human acute myeloid leukemia is organized as a hierarchy that originates from a primitive hematopoietic cell. Nat Med 3: 730737.

6. Yoshida GJ, Saya H (2012) [Therapeutic strategy for breast cancer stem cells] Nihon Rinsho 70: 243-248.

7. Yoshida GJ, Fuchimoto Y, Osumi T, Shimada H, Hosaka S, et al. (2012) LiFraumeni syndrome with simultaneous osteosarcoma and liver cancer: increased expression of a CD44 variant isoform after chemotherapy. BMC Cancer 12: 444.

8. Ghiaur G, Gerber J, Jones RJ (2012) Concise review: Cancer stem cells and minimal residual disease. Stem Cells 30: 89-93.

9. Acloque H, Adams MS, Fishwick K, Bronner-Fraser M, Nieto MA (2009) Epithelial-mesenchymal transitions: the importance of changing cell state in development and disease. J Clin Invest 119: 1438-1449.

10. Yoshida GJ, Saya H (2013) Inversed relationship between CD44 variant and c-Myc due to oxidative stress-induced canonical Wnt activation. Biochem Biophys Res Commun

11. Mani SA, Guo W, Liao MJ, Eaton EN, Ayyanan A, et al. (2008) The epithelialmesenchymal transition generates cells with properties of stem cells. Cell 133 704-715.

12. Goodison S, Urquidi V, Tarin D (1999) CD44 cell adhesion molecules. Mo Pathol 52: 189-196.

13. Brown RL, Reinke LM, Damerow MS, Perez D, Chodosh LA, et al. (2011) CD44 splice isoform switching in human and mouse epithelium is essential for epithelial-mesenchymal transition and breast cancer progression. J Clin Invest 121: 1064-1074.

14. Saint-André V, Batsché E, Rachez C, Muchardt C (2011) Histone H3 lysine 9 trimethylation and HP1y favor inclusion of alternative exons. Nat Struct $\mathrm{Mol}$ Biol 18: 337-344.

15. Yae T, Tsuchihashi K, Ishimoto T, Motohara T, Yoshikawa M, et al. (2012) Alternative splicing of CD44 mRNA by ESRP1 enhances lung colonization of metastatic cancer cell. Nat Commun 3: 883

16. Mani SA, Yang J, Brooks M, Schwaninger G, Zhou A, et al. (2007) Mesenchyme Forkhead 1 (FOXC2) plays a key role in metastasis and is associated with aggressive basal-like breast cancers. Proc Natl Acad Sci U S A 104: 1006910074.

17. Vandewalle C, Comijn J, De Craene B, Vermassen P, Bruyneel E, et al. (2005) SIP1/ZEB2 induces EMT by repressing genes of different epithelial cell-cell junctions. Nucleic Acids Res 33: 6566-6578.

18. Ocaña OH, Córcoles R, Fabra A, Moreno-Bueno G, Acloque H, et al. (2012) Metastatic colonization requires the repression of the epithelial-mesenchyma transition inducer Prrx1. Cancer Cell 22: 709-724.

19. Tsuji T, Ibaragi S, Hu GF (2009) Epithelial-mesenchymal transition and cell cooperativity in metastasis. Cancer Res 69: 7135-7139.

20. Yoshida GJ, Saya H, Zouboulis CC (2013) Three-dimensional culture of sebaceous gland cells revealing the role of prostaglandin E2-induced activation of canonical Wnt signaling. Biochem Biophys Res Commun 438: 640-646.

21. Roesch A, Fukunaga-Kalabis M, Schmidt EC, Zabierowski SE, Brafford PA, et al. (2010) A temporarily distinct subpopulation of slow-cycling melanoma cells is required for continuous tumor growth. Cell 141: 583-594.

22. Orian-Rousseau $V(2010)$ CD44, a therapeutic target for metastasising tumours. Eur J Cancer 46: 1271-1277.

23. Ishimoto T, Nagano O, Yae T, Tamada M, Motohara T, et al. (2011) CD44 variant regulates redox status in cancer cells by stabilizing the $\mathrm{xCT}$ subunit of system xc(-) and thereby promotes tumor growth. Cancer Cell 19: 387-400.

24. Cosentino-Gomes D, Rocco-Machado N, Meyer-Fernandes JR (2012) Cel Signaling through Protein Kinase C Oxidation and Activation. Int J Mol Sci 13 10697-10721. 
Citation: Yoshida GY, Saya H (2014) The Novel Anti-tumor Therapy Targeting the "Functional" Cancer Stem Cell Markers. Clin Exp Pharmacol 4: 147. doi:10.4172/2161-1459.1000147

25. Lambeth JD, Neish AS (2013) Nox Enzymes and New Thinking on Reactive Oxygen: A Double-Edged Sword Revisited. Annu Rev Pathol.

26. Wu Y, Zhang $X$, Kang X (2013) Oxidative stress inhibits adhesion and transendothelial migration, and induces apoptosis and senescence of induced pluripotent stem cells. Clinical and Experimental Pharmacology \& Physiology 40: 626-634.

27. Savaskan NE, Heckel A, Hahnen E, Engelhorn T, Doerfler A, et al. (2008) Small interfering RNA-mediated $\mathrm{xCT}$ silencing in gliomas inhibits neurodegeneration and alleviates brain edema. Nat Med 14: 629-632.

28. Timmerman LA, Holton T, Yuneva M, Louie RJ, Padró M, et al. (2013) Glutamine sensitivity analysis identifies the $\mathrm{XCT}$ antiporter as a common triple-negative breast tumor therapeutic target. Cancer Cell 24: 450-465.

29. Yoshikawa M, Tsuchihashi K, Ishimoto T, Yae T, Motohara T, et al. (2013) xCT inhibition depletes CD44v-expressing tumor cells that are resistant to EGFRtargeted therapy in head and neck squamous cell carcinoma. Cancer Res 73: 1855-1866.

30. Weissenstein U, Schumann A, Reif M, Link S, Toffol-Schmidt UD, et al. (2012) Detection of circulating tumor cells in blood of metastatic breast cancer patients using a combination of cytokeratin and EpCAM antibodies. BMC Cancer 12: 206
31. Gorges TM, Tinhofer I, Drosch M, Röse L, Zollner TM, et al. (2012) Circulating tumour cells escape from EpCAM-based detection due to epithelial-tomesenchymal transition. BMC Cancer 12: 178.

32. Tsikouras P, Tsagias N, Pinidis P, Csorba R, Vrachnis N, et al. (2013) The contribution of catumaxomab in the treatment of malignant ascites in patients with ovarian cancer: a review of the literature. Arch Gynecol Obstet 288: 581585.

33. Yoshida GJ, Saya H (2014) EpCAM expression in the prostate cancer makes the difference in the response to growth factors. Biochem Biophys Res Commun 443: 239-245.

34. Xu D, Hemler ME (2005) Metabolic activation-related CD147-CD98 complex. Mol Cell Proteomics 4: 1061-1071.

35. Patel M, Dalvi P, Gokulgandhi M (2013) Functional characterization and molecular expression of large neutral amino acid transporter (LAT1) in human prostate cancer cells. International Journal of Pharmaceutics 443: 245-253. 